\title{
DRAGON2: new features on energy losses treatment
}

\section{Arianna Ligorini ${ }^{\star a}$ Carmelo Evoli $^{b}$, Daniele Gaggero $c$, Andrea Vittino ${ }^{d}$, Giuseppe Di Bernardo $^{e}$, Mattia Di Mauro ${ }^{f}$, Piero Ullio ${ }^{g}$ and Dario Grasso ${ }^{h}$}

${ }^{a}$ Institute of Nuclear Physics - Polish Academy of Science, ul. Radzikowskiego 152, 31-342

Kraków, Poland

${ }^{b}$ Gran Sasso Science Institute, viale Francesco Crispi 7, 67100 L'Aquila (AQ), Italy

${ }^{c}$ GRAPPA Institute, University of Amsterdam, Science Park 904, 1090 GL Amsterdam, The Netherlands

${ }^{d}$ Physik-Department T30d, Technische Universität München, James-Franck-Straße 1, D-85748

Garching, Germany

${ }^{e}$ Max-Planck-Institut für Astrophysik, Karl-Schwarzschild-Straße 1, 85740 Garching bei München, Germany

$f$ 'W. W. Hansen' Experimental Physics Laboratory, Kavli Institute for Particle Astrophysics and Cosmology, Department of Physics and SLAC National Accelerator Laboratory, Stanford University, Stanford, CA 94305, USA

${ }^{g}$ Scuola Internazionale di Studi Superiori Avanzati, via Bonomea 265, 34136 Trieste, Italy

${ }^{h}$ INFN and Dipartimento di Fisica 'E. Fermi', Pisa University, Largo B. Pontecorvo 3, I-56127

Pisa, Italy

E-mail: arianna.ligorini@ifj.edu.pl, carmelo.evoli@gssi.infn.it,

d.gaggero@uva.nl, andrea.vittino@tum.de,

bernardo@mpa-garching.mpg.de, mailto:mdimauro@slac.stanford.edu, piero.ullio@sissa.it, dario.grassolpi.infn.it

In recent years we witnessed several experiments measuring a large set of observables related to Cosmic-ray physics with an unprecedented level of precision. In order to be able to fully exploit this great amount of new data we must act to refine our theoretical predictions. This can be achieved by building more realistic models of Cosmic-ray Galactic transport. The DRAGON project has been pursued in order to model Cosmic-rays propagation under realistic conditions and to allow a comparison with a wide set of experimental data. Studies brought forth with DRAGON showed how a treatment of Cosmic rays energy losses as realistic as possible is pivotal. In DRAGON2, the new version of the code, a more accurate, second order scheme for Cosmic Rays energy losses is implemented. In addition, the new version of the code allows us to investigate the impact of different models for Interstellar Radiation Field or galactic magnetic field. We present comparison between the previous energy losses approach and the new one, as well as validation test by comparing our numerical results with a set of analytical solutions. We study in particular the interplay of diffusion, reacceleration, and energy losses in a realistic case, and their impact on leptonic spectrum.

35th International Cosmic Ray Conference - ICRC2017

10-20 July, 2017

Bexco, Busan, Korea

${ }^{*}$ Speaker. 


\section{Introduction}

In recent years, a huge experimental effort has been brought forth in order to obtain new, very precise measurements of the high-energy cosmic radiation. Concerning charged cosmic-ray (CR) species, the bulk of results was provided by PAMELA orbital observatory, which also discovered several anomalies in the proton, helium and positron spectrum [1] and AMS-02; the latter operated on board of the International Space Station, and was able to confirm some of PAMELA results and obtaining unprecedented high-accuracy measurements up to the TeV [2] These results will soon be supplemented by measurements by CALET [3] and ISS-CREAM [4] (balloon borne experiments) as well as ground based KASCADE-Grande [5], and ARGO-YBG [6]. Concerning gamma ray radiation, measurement from HAWC [7] and CTA [8] should soon extend FERMI [9]results to higher energy. In the radio and microwave bands new results are expected by lofar by LOFAR [10] and SKA [11], after the recent probing by PLANK [12]. Finally, neutrino astronomy will be further investigated by IceCube [13] and KM3NeT [14], and Baikal-GVD [15] experiments.

DRAGON2 is the new version of the DRAGON code, a public software package designed to study CR propagation in the Galaxy. It was developed keeping in mind the need of a more accurate treatment of the many processes taking place during CR propagation.

Our presentation will be based on [16] and it will be organized as follows: in Section 2 we will introduce CRs propagation in the Galaxy, while Section 3 will describe the numerical approach we deploy to solve the transport equation. Section 4 will focus on energy losses (EL) treatment by presenting a numerical test and a comparison with the previous DRAGON code implementation. Section 5 will investigate the role of interplay between EL, reacceleration and diffusion mechanism, as well as momentum boundary condition. Section 6 will then contain our conclusions.

\section{Cosmic-ray transport in the Galaxy}

Many processes are relevant for CRs transport from Galactic acceleration sites to Earth: in particular, spatial and momentum diffusion, EL, advection, nuclear spallations and decays. The combination of all these processes can be described by the transport equation [17]. Assuming a steady state (i.e. $\partial N / \partial t=0$ ) the equation has the form:

$$
\begin{aligned}
& \nabla \cdot\left(\vec{J}_{i}-\vec{v}_{w} N_{i}\right)+\frac{\partial}{\partial p}\left[p^{2} D_{p p} \frac{\partial}{\partial p}\left(\frac{N_{i}}{p^{2}}\right)\right]-\frac{\partial}{\partial p}\left[\dot{p} N_{i}-\frac{p}{3}\left(\vec{\nabla} \cdot \vec{v}_{w}\right) N_{i}\right]= \\
& Q+\sum_{i<j}\left(c \beta n_{\mathrm{gas}} \sigma_{j \rightarrow i}+\frac{1}{\gamma \tau_{j \rightarrow i}}\right) N_{j}-\left(c \beta n_{\mathrm{gas}} \sigma_{i}+\frac{1}{\gamma \tau_{i}}\right) N_{i}
\end{aligned}
$$

where $N_{i}(\vec{r}, p)$ is the density (per momentum $p$ ) of the CR species $i . J_{i}=-D_{i k} \nabla_{k} N$ is the CR current associated to the process of CR spatial diffusion, modelled by means of the diffusion tensor $D_{i j}$, while $\vec{v}_{w}(\vec{r})$ is the Galactic wind velocity responsible for CR advection. $D_{p p}(\vec{r}, p)$ describes diffusion in momentum space (i.e. reacceleration), and $\dot{p}(\vec{r}, p)$ is the EL term. $Q(\vec{r}, p)$ is a source term, describing spatial distribution and energy spectra of sources. The timescale for radioactive decay at rest is given by $\tau_{i}$, while $\sigma_{i}$ is the spallation cross-section with interstellar gas of density $n_{\text {gas }}$. In the following we will neglect both spallation processes and radioactive decays, and the 
implementation of such processes in the code will be addressed in a forthcoming publication. The transport quantities can be either inferred from independent observations (e.g. gas distribution and magnetic field, which play a role in the loss term) or fitted to the data (e.g. diffusion coefficient, Galactic wind velocity). For all of them, different parameterizations are provided in literature. We implemented in DRAGON2 several options for the relevant transport quantities, as extensively described in Appendix $\mathrm{C}$ of [16]; in most cases, the quantities are position-dependent.

\subsection{Energy losses: a remark}

Regarding the specifical topic of this presentation, i.e. EL, we implemented in DRAGON2 all the processes that play a role for both nucleons (ionization, Coulomb interactions with the interstellar gas and pion production) and leptonic CRs (also inverse Compton, synchrotron emission and bremsstrahlung). A detailed review of all these processes with many references to literature may be found in Appendix C.10 of [16]. We would like to remark that the implementation of pion production as a continuous loss term for nucleons (in addition to ionisation and Coulomb losses) it's a novelty introduced in DRAGON2 with respect to earlier versions. The implementation of the pion production EL term followed [18]. We therefore performed numerical tests to evaluate the impact of pion-production EL term on the proton flux: this impact ranges from 5\% at $100 \mathrm{MeV}$ down to few percent at energies larger than $100 \mathrm{GeV}$. The impact on the proton slope is $0.5 \%$ [16].

Figure 1 presents a comparison of the EL time for all the mechanisms implemented in DRAGON2.

\section{Numerical approach}

\subsection{Solution of the transport equation}

A well-known approach to find the solution of a diffusive-advection equation is the operator splitting method.

The basic idea of this algorithm is to consider the transport equation 2.2 as a linear sum of different evolution operators (e.g., radial diffusion, vertical advection, EL, ...):

$$
\frac{\partial N}{\partial t}=\sum_{l} \mathscr{L}_{l}(N)+Q
$$

and for each of them to find a valid differencing scheme for updating $N$ from timestep $n$ to timestep $n+1$, considering only the single particular operator on the right-hand side of 3.1. The overall evolution in the time step $\Delta t$ is obtained by using separately all the operators in sequence( Local One Dimensional operator splitting).

The first step to solve Eq. 3.1 consists then into discretise the equation, i.e. write it on a discrete grid and transform derivative operators into finite differences. We consider a grid with spatial coordinates (indexes $i, \ldots, k$ ) and one momentum coordinate (index $q$ ); the grid spacing is arbitrary and it may be irregular. At a given point of this grid, the CR density and the source term can be written as $N_{i, \ldots, k, q}^{n}$ and $Q_{i, \ldots, k, q}^{n}$ where $n$ is the time-step index. To discretize the operators $\mathscr{L}_{l}$ we adopt finite difference approximations (in particular, we mainly use the centred difference scheme, which is known to give a truncation error $O\left(\Delta x^{2}\right)$ and $O(\Delta x)$ for uniform and non-uniform grids, respectively): 

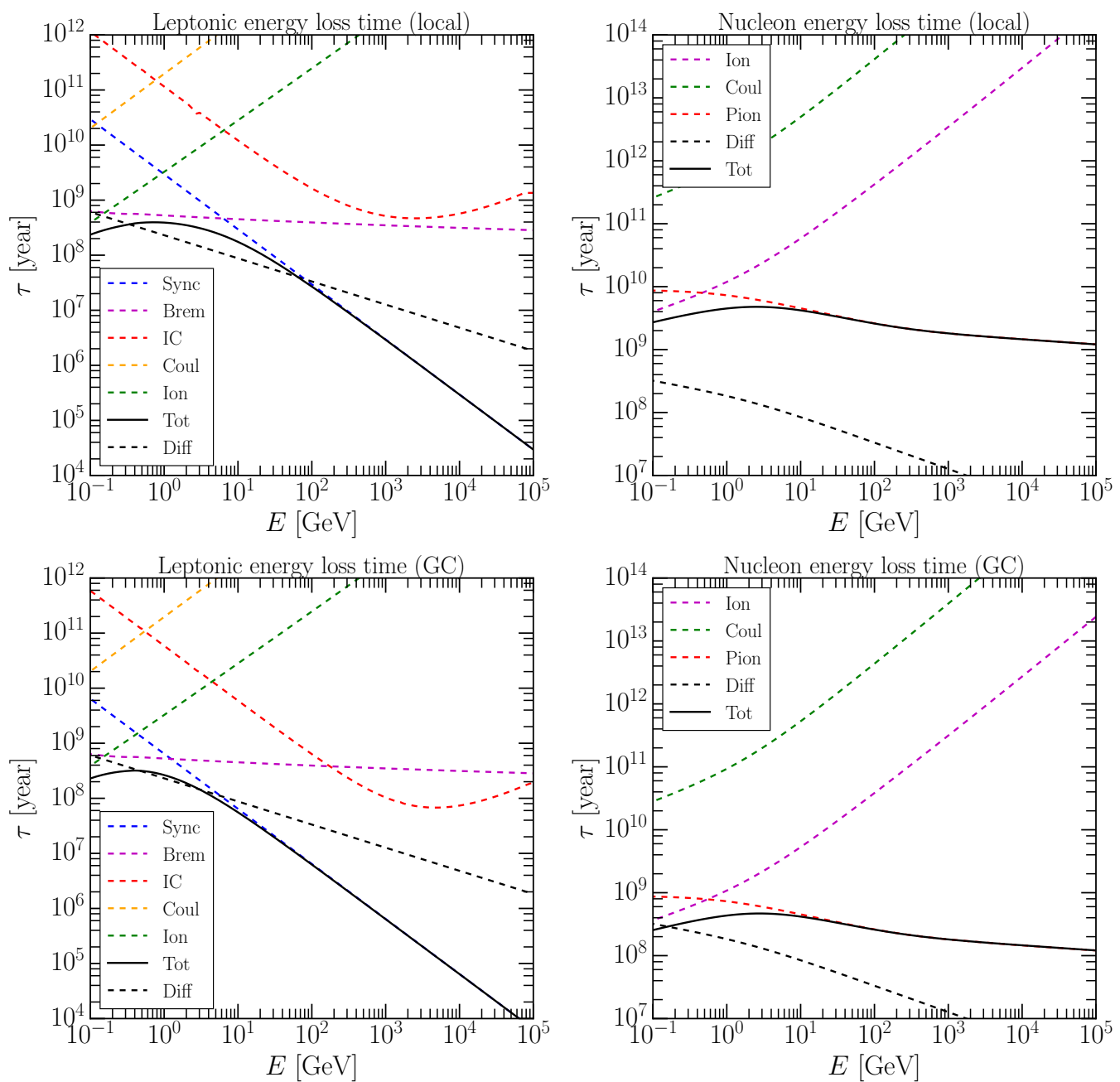

Figure 1: The EL timescales for electrons or positrons (left panels) and protons (right panels) are shown for the mechanisms implemented in DRAGON2. (Local gas density $n_{\mathrm{H}}=0.9 \mathrm{~cm}^{-3}$ (top), $\mathrm{GC} n_{\mathrm{H}}=10 \mathrm{~cm}^{-3}$ (bottom); constant ISRF from [19], magnetic field model from [20]). Black dashed line is diffusion timescale.

$$
\begin{aligned}
\frac{\partial N}{\partial x} & \rightarrow \frac{N_{i+1}-N_{i-1}}{x_{i+1}-x_{i-1}} \\
\frac{\partial^{2} N}{\partial x^{2}} & \rightarrow \frac{2}{x_{i+1}-x_{i-1}}\left(\frac{N_{i+1}-N_{i}}{x_{i+1}-x_{i}}-\frac{N_{i}-N_{i-1}}{x_{i}-x_{i-1}}\right)
\end{aligned}
$$

The second step in finding a solution consists in defining a rule to compute $N^{n+1}$ from $N^{n}$. In DRAGON2 this is mostly realised through the Crank-Nicolson (CN) method [21], defined as follows:

$$
\frac{N_{i}^{n+1}-N_{i}^{n}}{\Delta t}=\frac{1}{2}\left[\hat{\mathscr{L}}_{i}^{n+1}+\hat{\mathscr{L}}_{i}^{n}\right]+\frac{Q_{i}}{n_{\mathrm{os}}}
$$

where $\Delta t$ is the time-interval between the time-steps and $n_{\mathrm{os}}$ is the number of operators that appear in Eq. 3.1. 


\subsubsection{The energy loss operator}

To improve our treatment of EL in the new version of the code, we choose to adopt for the EL a 2-nd order accurate method which is obtained by integrating in momentum the EL term and evaluating the integral with the trapezoidal rule. A similar approach was already implemented in the PICARD code and it's described in detail in [22].

In Section 4.1, we compare the performances of this scheme with the 1-st order discretisation scheme, as implemented in the previous version of DRAGON.

\section{Validation of solver and numerical tests}

When checking the validity of the solver, the intent is to investigate both the convergence and the accuracy of the numerical approach that we use. Various tests have been performed on DRAGON2, and the complete set of results can be found in [16].

In a steady-state condition, the transport equation 3.1 can be rewritten as: $\mathscr{L}(\vec{x}, p)+Q(\vec{x}, p)=0$. One can then introduce the residual $\|\mathscr{R}\|^{2}$ as:

$$
\|\mathscr{R}\|^{2}=\frac{1}{N_{Q}} \sum_{i}\left(\frac{\hat{\mathscr{L}}_{i}+Q_{i}}{Q_{i}}\right)^{2}
$$

where $N_{Q}$ is the number of grid points where the condition $Q \neq 0$ is satisfied. During the iteration procedure, the residual decreases and finally converges to a constant value which depends on the grid resolution and on the adopted discretisation scheme.

We then run tests under simplified assumptions to compare the numerical scheme of each transport operator against an analytical solution. We quantify accuracy by means of the relative error $\varepsilon_{\text {rel }}$ :

$$
\varepsilon_{\mathrm{rel}}=\max \left(\frac{N_{i}^{a}-N_{i}^{m}}{N_{i}^{a}}\right)
$$

where $N_{i}^{a}$ is the analytical solution evaluated at the grid point corresponding to the index $i$, and $N_{i}^{m}$ is the corresponding numerical solution as obtained after convergence has been attained.

\subsection{Test for energy loss operator and comparison with DRAGON}

A simplified, steady-state transport equation taking only EL and source terms into account can be written as: $\frac{\partial}{\partial p}(\dot{p} N)=Q(p)$.

The analytic solution this equation can be found in Appendix B.3 of [16].

In the upper panels of Fig. 2 we comparethe analytical solution with the numerical one as obtained for $\Delta t=1 \mathrm{Myr}$ with the 1-st and the 2-nd order discretisation schemes; in the lover panels we show absolute and relative errors at $p=10 \mathrm{GeV}$ as a function of the grid size. With 32 grid points the 1-st order scheme produces a $\sim 25 \%$ error with respect to the analytical solution, while the 2-nd order scheme accounts for a $\sim 2.5 \%$ error only. Expected scalings of the relative error are correctly reproduced by the numerical solution. Remarkably, the 2-nd order scheme provides a $\lesssim 10^{-2}$ accuracy already with 64 grid points, while the same accuracy is reached by the first order scheme with more than 512 points. 

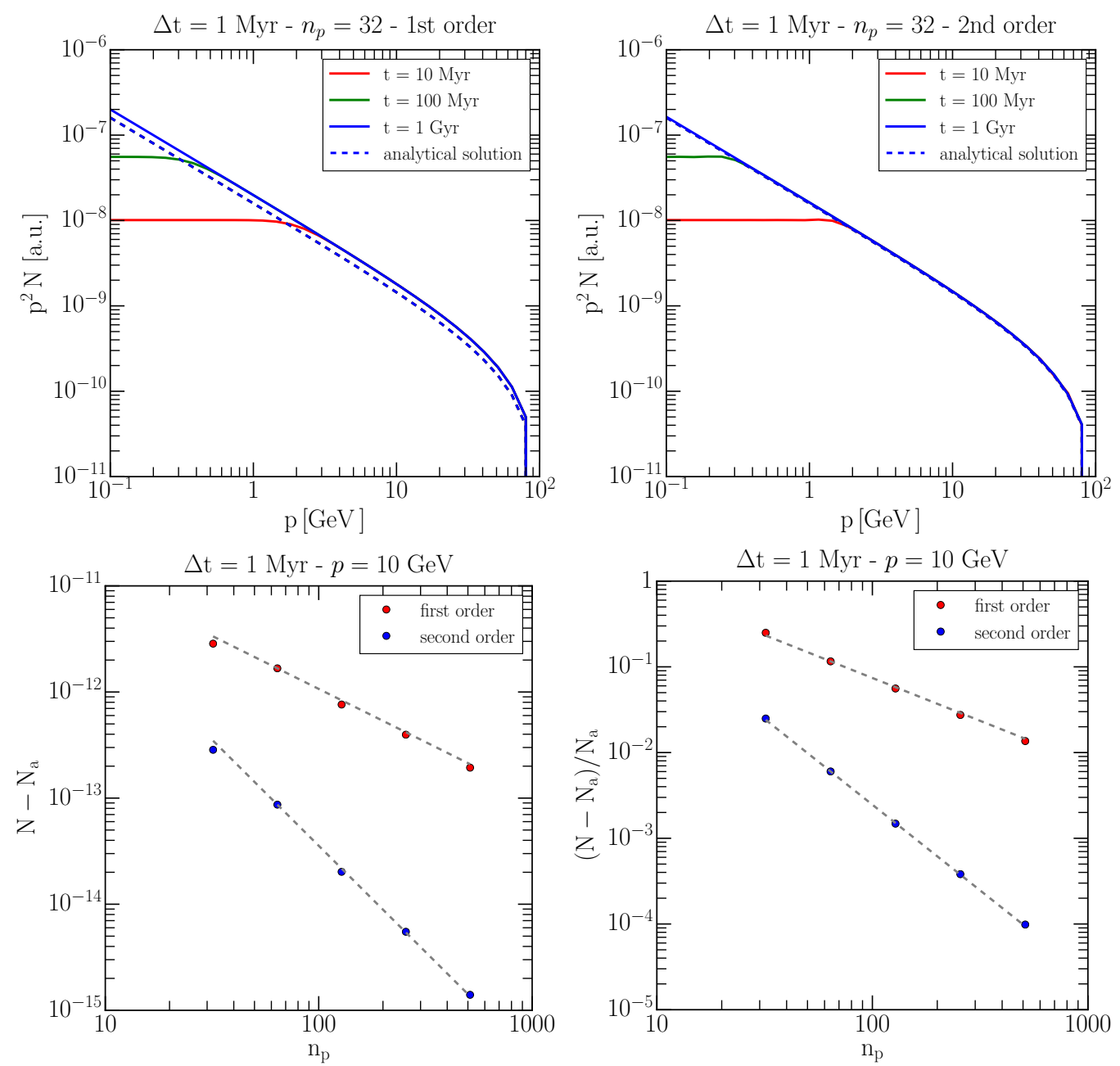

Figure 2: Top: Comparison between the numerical and analytical solution for $\Delta t=1 \mathrm{Myr}$ in both the 1-st (left panel) and 2-nd order (right panel) cases, for EL term. Bottom: Absolute (left panel) and relative error (right panel) scaling with the number of grid points, for both 1-st and 2-nd order schemes.

\section{Interplay of diffusion, reacceleration, and leptonic energy losses: the role of the boundary condition in momentum}

Leptonic spectrum is significantly shaped by EL due to synchrotron emission and inverse Compton scattering. We considerhere a realistic setup in which the EL term is coupled with reacceleration and diffusion operators.

A significant reacceleration, in combination with EL, produces a hump-like feature in the spectrum [16]. In Fig. 3 we show the CR spectrum at the Sun position, and we compare the performance ofDRAGON2 solver with the one implemented in the first version of DRAGON. The hump is clearly visible in the spectrum in both cases, and give rise to a peak at $2.5 \mathrm{GeV}$ : this feature is caused by the interplay between the two competing effects of EL (responsible for a downwards flux in momentum space) and reacceleration (which is a diffusive term in momentum space, and is responsible 

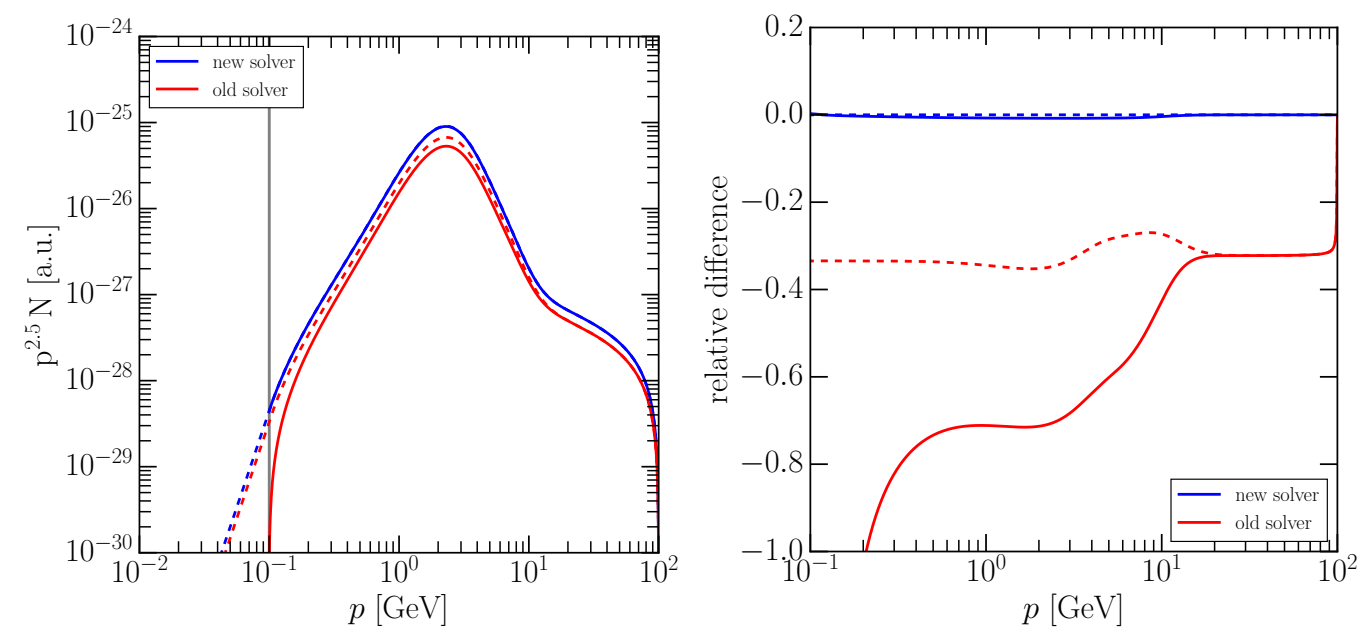

Figure 3: Left panel: CR spectrum at the Sun position in arbitrary units. Red line: DRAGON solver; blue line: DRAGON2 solver. In both cases the CR injection is modelled as a power-law starting from $p_{i n j}^{*}=0.1$ $\mathrm{GeV}$ (grey vertical line). Dashed lines refer to the simulation momentum boundary set at $p_{\min }=0.1 p_{\text {inj }}^{*}$, solid line to $p_{\min }=p_{i n j}^{*}$. Right panel: relative difference with respect to the new solver, line legend as in the left panel.

for an upwards flux ).

In DRAGON solver the boundary conditions in momentum are obtained by imposing null derivatives for the CN coefficients. DRAGON2 solver boundary conditions are defined by[16] $\frac{\partial}{\partial p}\left(\frac{N}{p^{2}}\right)_{p_{\text {min }}}=0$.

We set the minimum momentum of CR injection at $p_{i n j}^{*}=0.1 \mathrm{GeV}$ and the boundary of the simulation at $p_{\min }=0.1 p_{i n j}^{*}$. In this setup, the two solvers exhibit a $30 \%$ difference at all energies due to the better accuracy of the 2nd-order scheme. We then compare each solver with the case in which $p_{\min }=p_{i n j}^{*}$. We notice that DRAGON2 solution is practically unchanged, while DRAGON solution is significantly different at lower momenta.

\section{Conclusions}

We presented DRAGON2, the new version of DRAGON code. We discussed here the new setup for treating EL: it is evident the better accuracy of the new 2-nd order approach. We remark that this improvement in accuracy is obtained without a significant increase in the number of iterations required to reach convergence.. We also checked thatDRAGON2 appears to be more accurate in reproducing the expected feature due to interplay of EL, reacceleration and diffusion. Also, changing the simulation momentum boundary causes different effect with the two solvers, due to different ways of imposing momentum boundary condition: the momentum boundary condition of DRAGON2 enables us to impose $p_{\min }=0.1 p_{i n j}^{*}$ with negligible effect on the solution accuracy.

\section{References}

[1] P. Picozza, et al., PAMELA: A Payload for Antimatter Matter Exploration and Light-nuclei Astrophysics, Astropart. Phys. 27 (2007) 296-315 [astro-ph/ 0608697 ]. 
[2] A. Kounine et al., The Alpha Magnetic Spectrometer on the International Space Station, Int. J. Mod. Phys. E21 (08)(2012)1230005.

[3] S. Torii, The CALET mission for detection of cosmic ray sources and dark matter, J. Phys. Conf. Ser 120 (17) (2008) 062020.

[4] E. S. Seo et al., Cosmic Ray Energetics And Mass for the International Space Station (ISS-CREAM),Adv. Space Res. 53 (2014) 1451-1455.

[5] T. Antoni et al., The Cosmic ray experiment KASCADE, Nucl. Instrum. Meth. A513 (2003) 490-510.

[6] G. Aielli et al., Layout and performance of RPCs used in the Argo-YBJ experiment, Nucl. Instrum. Meth. A562 (2006) 92-96

[7] A. J. Smith, HAWC: Design, Operation, Reconstruction and Analysis, ArXiv e-prints (Aug., 2015)[1508.05826].

[8] M. Actis et al., Design concepts for the Cherenkov Telescope Array CTA: An advanced facility for ground-based high-energy gamma-ray astronomy, Exper. Astron. 32 (2011) 193-316, [1008 . 3703 ].

[9] W. B. Atwood et al., The Large Area Telescope on the Fermi Gamma-Ray Space Telescope Mission, ApJ 697 (2009) 1071-1102,[0902.1089].

[10] M. P. van Haarlem et al., LOFAR: The LOw-Frequency ARray, A\&A 556 (2013) A2, [1305. 3550 ].

[11] P. Quinn, et al., Delivering SKA Science, in prooceedings of AASKA 2014 POS (AASKA14) 147 (2015)

[12] The Planck Collaboration, The Scientific Programme of Planck, ArXiv e-prints (Apr., 2006) [astro-ph/0604069].

[13] M. G. Aartsen et al., Contributions to ICRC 2015 Part II: Atmospheric and Astrophysical Diffuse Neutrino Searches of All Flavors, in proceedings of ICRC 2015 [1510 . 05223]

[14] A. Margiotta, The KM3NeT deep-sea neutrino telescope, Nucl. Instrum. Meth. A766 (2014) 83-87, [1408.1392].

[15] A. D. Avrorin, et al., Baikal-GVD: first cluster Dubna in proceedings of EPS-HEP 2015 POS (EPS-HEP2015) 418, (2015).

[16] C. Evoli, D. Gaggero, A. Vittino, G. Di Bernardo, M. Di Mauro, A. Ligorini, P. Ullio, D. Grasso, Cosmic-ray propagation with DRAGON2: I. numerical solver and astrophysical ingredients, JCAP, 1702 (02) 015 (2017) [1607. 07886].

[17] V. L. Ginzburg and S. I. Syrovatskii, The Origin of Cosmic Rays 1964.

[18] S. Krakau and R. Schlickeiser, Pion Production Momentum Loss of Cosmic Ray Hadrons, ApJ 802 (2015) 114.

[19] T. Delahaye, et al., Galactic electrons and positrons at the Earth: new estimate of the primary and secondary fluxes, A\&A $\mathbf{5 2 4}$ (2010) A51, [1002 . 1910].

[20] X. H. Sun, et al., Radio observational constraints on Galactic 3D-emission models, A\&A 477 (2008) 573-592, [0711.1572].

[21] W. H. Press, et al., Numerical recipes in C++ : the art of scientific computing 2002.

[22] R. Kissmann, PICARD: A novel code for the Galactic Cosmic Ray propagation problem, Astropart. Phys. 55 (2014) 37-50, [1 401 . 4035$]$ 